\title{
HIV-activated human plasmacytoid DCs induce Tregs through an indoleamine 2,3-dioxygenase-dependent mechanism
}

\author{
Olivier Manches, ${ }^{1}$ David Munn, ${ }^{2}$ Anahita Fallahi, ${ }^{1}$ Jeffrey Lifson, ${ }^{3}$ \\ Laurence Chaperot, ${ }^{4,5}$ Joel Plumas, ${ }^{4,5}$ and Nina Bhardwaj ${ }^{1}$
}

\begin{abstract}
${ }^{1}$ NYU Cancer Institute, New York University, New York, New York, USA. ${ }^{2}$ Department of Pediatrics, Institute for Molecular Medicine and Genetics, Medical College of Georgia, Augusta, Georgia, USA. ${ }^{3}$ AIDS Vaccine Program, Science Applications International Corporation — Frederick Inc., National Cancer Institute, Frederick, Maryland, USA. ${ }^{4}$ INSERM U823, Immunobiologie et Immunothérapie des cancers, and EFS Rhône-Alpes, La Tronche, France. ${ }^{5}$ Université Joseph Fourier, Grenoble, France.
\end{abstract}

\begin{abstract}
Plasmacytoid DCs (pDCs) have been implicated as crucial cells in antiviral immune responses. On recognizing HIV, they become activated, secreting large amounts of IFN- $\alpha$ and inflammatory cytokines, thereby potentiating innate and adaptive antiviral immune responses. Here, we have shown that HIV-stimulated human pDCs can also induce the differentiation of naive $\mathrm{CD}^{+} \mathrm{T}$ cells into Tregs with suppressive function. This differentiation was independent of $\mathrm{pDC}$ production of IFN- $\alpha$ and primarily dependent on $\mathrm{pDC}$ expression of indoleamine 2,3-dioxygenase, which was induced through the TLR/MyD88 pathway, following binding of HIV to CD4 and triggering of TLR7 by HIV genomic RNA. Functionally, the Tregs induced by pDCs were shown to inhibit the maturation of bystander conventional DCs. This study therefore reveals what we believe to be a novel mechanism by which PDC may regulate and potentially limit anti-HIV immune responses.
\end{abstract}

\section{Introduction}

Plasmacytoid DCs ( $\mathrm{pDCs}$ ) constitute a crucial subset of APCs involved in the antiviral immune response. Human $\mathrm{pDCs}$ express the pattern recognition receptors TLR7 and TLR9, allowing them to respond to virus-associated single-stranded RNA and unmethylated CPG DNA, respectively, and are the main source of type I interferon upon viral infection. pDCs are believed to play an important role in recognition of HIV-1, as they secrete high amounts of IFN- $\alpha(1,2)$ and other immune mediators in response to infection (1). HIV recognition by pDCs is likely mediated by the innate receptor TLR7 and requires its endocytosis followed by endosomal acidification (1). Despite the ability of HIV to activate pDCs, the number of circulating pDCs, as well as conventional DCs (cDCs), decreases during primary as well as chronic HIV infection, perhaps due to migration to infection sites and/or apoptosis (3). In contrast to pDCs, cDCs that also express TLR7 are not activated by HIV. However, HIV-activated pDCs (HIV-pDCs) can induce bystander maturation of $\mathrm{cDCs}$ through the secretion of IFN- $\alpha$ and TNF- $\alpha$ (2), suggesting a role for pDCs in regulating the maturation of and possibly antigen presentation by cDCs.

The role of pDCS in adaptive immunity, and particularly their capacity to present antigen, is still debated. It has been suggested recently that human $\mathrm{pDCs}$ can cross-present HIV antigens to CD8 ${ }^{+}$ T cells (4). In other studies, pDCs were shown to induce either Th1 or Th2 responses from naive $\mathrm{CD} 4^{+} \mathrm{T}$ cells $(2,5,6)$, depending upon the stimulus. In contrast, several studies have documented a role

Nonstandard abbreviations used: AT-2 HIV, aldrithiol-2-inactivated HIV; cDC, conventional DC; COX-2, cyclooxygenase-2; CTLA-4, CTL antigen 4; GITR, glucocorticoid-induced TNF receptor; ICOS-L, inducible costimulatory molecule ligand; IDO, indoleamine 2,3-dioxygenase; MLR, mixed lymphocyte reaction; moDC, monocytederived DC; pDC, plasmacytoid DC.

Conflict of interest: N. Bhardwaj is a coinventor on patents pertaining to human dendritic cell preparations.

Citation for this article: J. Clin. Invest. 118:3431-3439 (2008). doi:10.1172/JCI34823. for activated $\mathrm{pDCs}$ in the induction of $\mathrm{CD}^{+}{ }^{+}$or $\mathrm{CD}^{+}$Tregs, both in vitro (7-10) and in vivo (11). For example, pDCs cultured in the presence of IL-3 and activated by CD40L induce the differentiation of $\mathrm{CD}^{+}(7)$ or $\mathrm{CD}^{+}$Tregs (8) from naive cells. Ligation of TLR9 by unmethylated $\mathrm{CpG}$ oligonucleotides activates $\mathrm{pDCs}$ and can also induce the generation of $\mathrm{CD}^{+}$Tregs $(8,10)$. A recent study in mice suggests that $\mathrm{pDCs}$ can mediate the tolerization of alloantigenspecific T cells by inducing Foxp $3^{+}$Tregs in vivo (11). Finally, viral activation of $\mathrm{pDCs}$ by herpes simplex virus 1 (HSV1) leads them to induce allogeneic $\mathrm{CD}^{+} \mathrm{T}$ cells to differentiate into cytotoxic Tregs, a phenomenon that is partially dependent on IL-10 and inducible costimulatory molecule ligand (ICOS-L) (9). These data indicate that $\mathrm{pDCs}$ have an inherent plasticity, inducing a diversity of responses that are partially stimulus dependent. Apparently, pDCs limit the induction of overly intense immune responses in certain situations through the generation of immunoregulatory Tregs.

Immune activation in HIV has been associated with expression of the activation marker CD38 on $\mathrm{CD}^{+} \mathrm{T}$ cells, and a direct correlation between $\mathrm{CD}^{+} \mathrm{T}$ cell activation levels and disease progression levels has been confirmed in many studies (12-14). We investigated whether HIV-stimulated pDCs can regulate the levels of immune activation by promoting the differentiation of regulatory $\mathrm{CD}^{+} \mathrm{T}$ cells. In this study, we show for the first time to our knowledge that pDCs induce the development of Tregs from naive $\mathrm{CD}^{+} \mathrm{T}$ cells, primarily through expression of indoleamine 2,3 dioxygenase (IDO), a component of the enzymatic pathway involved in the catabolism of tryptophan. Tryptophan depletion and generation of immunoactive kynurenine metabolites by IDO have been shown to suppress proliferation of activated $\mathrm{T}$ cells, and IDO expression by fetal tissues and tumors participates in the downregulation of immune activation in these settings $(15,16)$. Moreover, a recent study described IDO expression in pDCs of HIV-infected patients that was responsible for decreased mitogenic responses in their PBMCs (17). 
Our study uncovers what we believe to be a novel role of IDO expression in $\mathrm{pDCs}$ through the generation of Tregs that can suppress $\mathrm{CD}^{+} \mathrm{T}$ cell proliferation. We also demonstrate that pDC-induced Tregs can impair maturation of cDCs, potentially limiting their antigen presentation and thereby enhancing the overall suppressive effect of Tregs. Our data suggest that pDCs play a dual role, enhancing innate and adaptive immune responses to HIV, while simultaneously limiting the extent of immune activation through generation of Tregs.

\section{Results}

$H I V-p D C$ induce the generation of Tregs from naive $C D 4^{+} T$ cells. We first evaluated whether HIV-exposed pDCs could induce Tregs from naive $\mathrm{CD}^{+} \mathrm{T}$ cells. Purified $\mathrm{pDCs}$ (donor A) were activated for 2 days by Aldrithiol-2-inactivated HIV (AT-2 HIV), and the TLR agonists CPGB or resiquimod (R-848, a TLR7/8 agonist). They were subsequently added to allogeneic naive $\mathrm{CD} 4^{+} \mathrm{T}$ cells (donor B). After 7 days, responding $T$ cells were added to a primary mixed lymphocyte reaction (MLR) consisting of irradiated PBMCs (donor A, "stimulator") and naive CD4 $4^{+} \mathrm{T}$ cells (donor $\mathrm{B}$, "responder"). As shown in Figure $1 \mathrm{~A}, \mathrm{CD} 4^{+} \mathrm{T}$ cells stimulated with AT-2 HIV-activated pDCs displayed poor proliferative responses upon restimulation and could partially suppress the proliferation of naive $\mathrm{CD} 4^{+} \mathrm{T}$ cells in the MLR or block blast formation upon stimulation of naive $\mathrm{CD}^{+} \mathrm{T}$ cells (Supplemental Figure 1; supplemental material available online with this article; doi:10.1172/JCI34823DS1). Similar results were obtained regardless of whether the pDCs in the first culture were stimulated with AT-2 HIV, CpG, or resiquimod. In contrast, T cells stimulated during the first culture with unactivated $\mathrm{pDCs}$ did not show high proliferative responses upon restimulation, nor did they suppress the MLR response in the second culture.

$\mathrm{T}$ cells primed by HIV-pDCs inhibited the proliferation of naive $\mathrm{CD}^{+} \mathrm{T}$ cells in a dose-dependent fashion (Figure $1 \mathrm{~B}$ ) and contained a population of Foxp $3^{+} \mathrm{CD} 127^{\text {lo }}$ cells compatible with a Treg phenotype (Figure 1C) $(18,19)$. This population constituted less than $1 \%$ up to $15 \%$ of the whole population, depending on the experiment, thereby accounting for the fact that higher ratios of Tregs to naive $T$ cells were needed to achieve significant suppression. Foxp3 expression was most evident after day 5, when most Foxp $3^{+}$cells also expressed CD25. However, at later time points, a distinct population of cells expressing Foxp3 only remained (Supplemental Figure 2).

The pDC-primed $\mathrm{T}$ cell population could be further separated into CD25 hi and CD25 lo cells. Suppressive activity for the MLR was contained within the $\mathrm{CD} 25^{+}$population (Figure 1D). Transwell experiments showed that the Tregs generated by original HIV-pDCs inhibit naive CD4 ${ }^{+} \mathrm{T}$ cell proliferation in a contactdependent manner (Figure 1E).

The pDC line GEN2.2 is activated by HIV and artificial TLR agonists, similar to blood-derived pDCs. The human pDC line GEN2.2 (GEN) was described previously (20) and shares most phenotypic and functional attributes of normal pDCs. GEN cells secreted IFN- $\alpha$ in response to artificial TLR agonists and to HIV (Figure 2A), like primary pDCs. They mature phenotypically upon incubation with HIV or AT-2 HIV, as shown by upregulation of CD80 and CD40 (Figure 2, B and C) and CCR7 and CD83 (Supplemental Figure 5). Activation of GEN cells with HIV also induced Treg generation (Figure 2D), and such cells were used further as a model for studying Treg generation by HIV-pDCs, in parallel with primary pDCs.
Mechanisms of Treg suppression and generation. In order to investigate the mechanisms of Treg generation, known pathways implicated in the generation of Tregs were blocked using specific inhibitors and blocking antibodies. Blocking the type I interferon pathway with neutralizing antibodies against IFN- $\alpha$, IFN $-\beta$, and IFN- $\alpha / \beta$ receptor during $\mathrm{pDC}$ activation and culture with naive $\mathrm{T}$ cells did not inhibit the generation of Tregs (Figure $3 \mathrm{~A}$ ). As it has been shown that tumor-derived cyclooxygenase-2 (COX-2)/ $\mathrm{PGE}_{2}$ could modulate Foxp3 expression and Treg activity in some cancers (21), we examined whether blocking this pathway could inhibit Treg generation. Inhibiting the $\mathrm{COX}-2 / \mathrm{PGE}_{2}$ pathway by addition of EP2 and EP4 antagonists (AH1 and AH2) and indomethacin did not have any significant effect on the differentiation of Tregs (Figure 3B). Glucocorticoid-induced TNF receptor (GITR) and TGF- $\beta$ inhibition using blocking antibodies did not affect Treg generation, while neutralizing antibodies against IL-10, CTL antigen 4 (CTLA-4), and ICOS-L only partially inhibited Treg generation (Table 1 ).

Expression of IDO by DCs has been shown to confer tolerogenic stimuli in various settings (22-24), and it was recently shown that HIV-pDCs express functional levels of IDO (17). Indeed, activation of GEN cells by HIV or the TLR7/8 agonist resiquimod induced transcription of IDO (Figure 4A). IDO protein was also induced in primary pDCs by HIV and resiquimod (Figure 4B). In our hands, the fusion protein CTLA-4-Ig did not induce or enhance IDO expression by pDCs (Figure 4B), as it did not induce or enhance IDO expression and function by monocyte-derived DCs (moDCs) (Supplemental Figure 3). Adding the specific inhibitor 1-methyltryptophan during the Treg generation culture significantly inhibited the differentiation of Tregs (Figure 4C). Furthermore knockdown of the expression of IDO by siRNA in GEN cells prevented generation of Tregs (Figure 4D). Because recent studies showed that ICOS-L upregulation on pDCs was responsible for the generation of IL-10-secreting $\mathrm{T}$ cells with a suppressive activity (8), reduction of ICOS-L expression was also tested using specific siRNA in pDCs. Knockdown of ICOS-L expression by siRNA did not influence the suppressive activity of the $\mathrm{T}$ cells generated in the primary culture. siRNA-mediated knockdown of TLR7 and MyD88 (but not TLR9) also showed that IDO transcription is at least partially dependent on the TLR7/MyD88 pathway, which is activated by HIV in pDCs (Figure 4E) (1). In order to investigate the importance of viral entry and confirm the role of endosomal activation of TLR7 for IDO expression, blocking of the pathway was performed at 3 levels. Interaction of the viral envelope with CD4 was prevented by addition of a neutralizing anti-CD4 antibody and a human anti-gp120 antibody (b12) (1). As endosomal acidification/maturation is required for TLR7 signaling (25) and HIV activation of pDCs (1), it was inhibited using the weak base chloroquine. Finally, TLR7 signaling was blocked using the specific inhibitory oligonucleotide IRS 661 (26). As shown in Figure $4 \mathrm{~F}$, blocking endosomal acidification by chloroquine or TLR7 signaling by IRS 661 completely blocked HIV-induced expression of IDO, whereas blocking the CD4-envelope interaction almost completely abrogated IDO expression. Accordingly, secretion of IFN- $\alpha$ and TNF- $\alpha$ was also inhibited in these conditions (data not shown). Together, these data show that IDO is induced through activation of the TLR7/MyD88 pathway after viral entry and delivery into an acidic compartment.

Tregs generated by HIV-pDCs can suppress the phenotypic maturation of $c D C s$. It has been shown that peripheral CD $4^{+} C D 25^{\text {hi }}$ cells can inhibit the $\mathrm{cDC}$, but not the pDC, maturation program (27). 
A

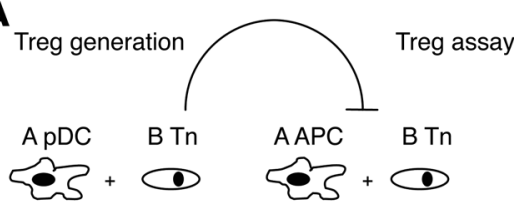

B
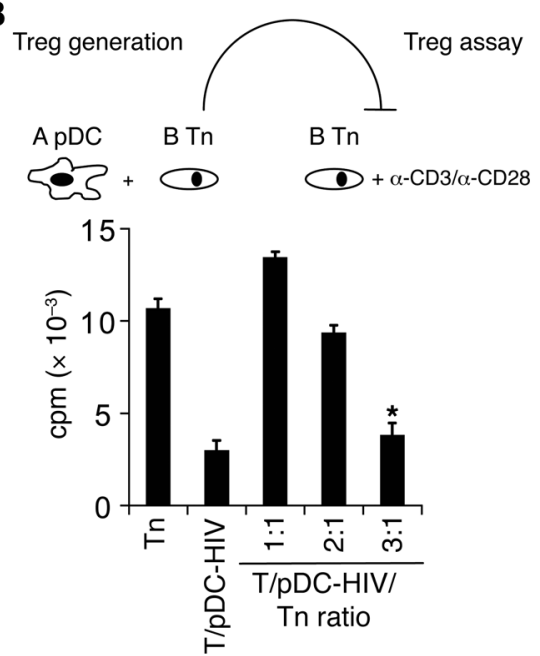

C

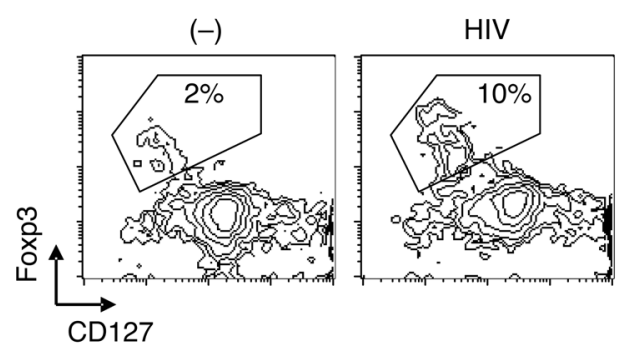

$\mathbf{E}$

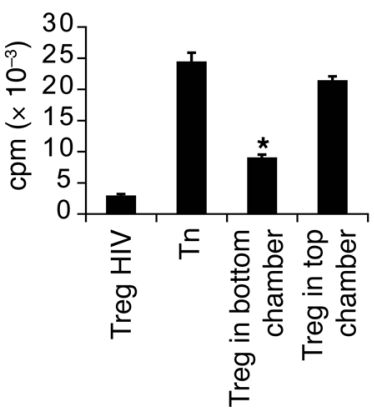

Figure 1

HIV-activated pDCs induce Tregs from naive CD4+ T cells. (A) AT-2 HIV- or CpGB- or resiquimod-activated pDCs (donor A) were incubated with naive $C D 4^{+} T$ cells (donor $B$ ) for 7 days. The responding $T$ cells were then added to a primary allogeneic MLR (the composition of which is displayed in the inset) using PBMCs (A APC) as stimulatory cells and naive CD4+ T cells (B Tn) as responding cells. "CD4" refers to the naive T cells alone; "CD4 + APC" refers to the MLR of the naive CD4+ $T$ cells to PBMCs. Proliferation was measured by thymidine incorporation after 5 days. ${ }^{*} P<0.01$, Student's $t$ test, compared with proliferation of naive T cells in the MLR (far-left black bar). Results shown are representative of 5 experiments. (B) pDCs were activated by AT-2 HIV and incubated with naive CD4+ ${ }^{+}$cells. The T cells (T/pDC-HIV) were then added at different ratios to naive $C D 4^{+} T$ cells stimulated with anti-CD3 and anti-CD28 antibodies, and proliferation (thymidine incorporation) was measured after 5 days. ${ }^{*} P<0.01$, Student's $t$ test, compared with proliferation of naive T cells alone. Results are representative of 3 experiments. (C) Phenotype of the day 7 T cell population generated by HIV-activated or unactivated pDCs, gated on CD4+ T cells. Expression of CD127 and Foxp3 is shown. Percentages of cells in each gate $\left(F o x p 3^{+} C D 127^{\circ}\right)$ are indicated. (D) T cells generated as in B were separated into CD25- and $\mathrm{CD}_{25}{ }^{+}$populations by magnetic bead enrichment and then added to naive $\mathrm{T}$ cells $(\mathrm{Tn})$ and stimulated as described in $\mathbf{B}$. Proliferation was measured after 5 days. Results are representative of more than 5 experiments. (E) T cells stimulated as in B were added to naive CD4 ${ }^{+} T$ cells in the upper chamber of a Transwell plate or in the same lower compartment as naive CD4+ $\mathrm{T}$ cells stimulated with anti-CD3 and anti-CD28 antibodies. Proliferation was measured after 5 days by thymidine incorporation. Results are representative of 3 experiments. ${ }^{*} P<0.01$, Student's $t$ test, compared with proliferation of naive T cells alone.

We therefore tested whether HIV-pDC-induced Tregs could also influence $\mathrm{pDC}$ and $\mathrm{cDC}$ maturation. pDCs were activated by CPG and moDCs by LPS or resiquimod, with or without preincubation with CD3-stimulated Tregs (Figure 5). As a control, $\mathrm{T}$ cells stimulated by anti-CD3/CD28 antibodies were added instead of Tregs. As shown in Figure 5A, Tregs and control T cells both tended to partially inhibit pDC maturation, as measured by $\mathrm{CD} 83$ and $\mathrm{CD} 40$ upregulation, although the inhibition did not reach statistical significance, and they both induced some level of activation in pDCs by themselves. On the contrary, neither subset of $\mathrm{T}$ cells matured moDCs in the absence of TLR stimulation (as measured by CD86 and CD83 upregulation). However, Tregs impaired maturation of moDCs induced by resiquimod (Figure 5B) and LPS (data not shown), whereas nonTregs were not inhibitory.

Together, these data demonstrate that HIV-pDCs can generate Treg from naive $\mathrm{CD} 4^{+} \mathrm{T}$ cells in a TLR7- and IDO-dependent manner. They also show that these Tregs can inhibit the maturation of cDCs, highlighting their complex role during HIV infection. pDCs that encounter HIV therefore not only help mount a vigorous innate immune response but can simultaneously restrict such responses through various Treg-dependent mechanisms. 

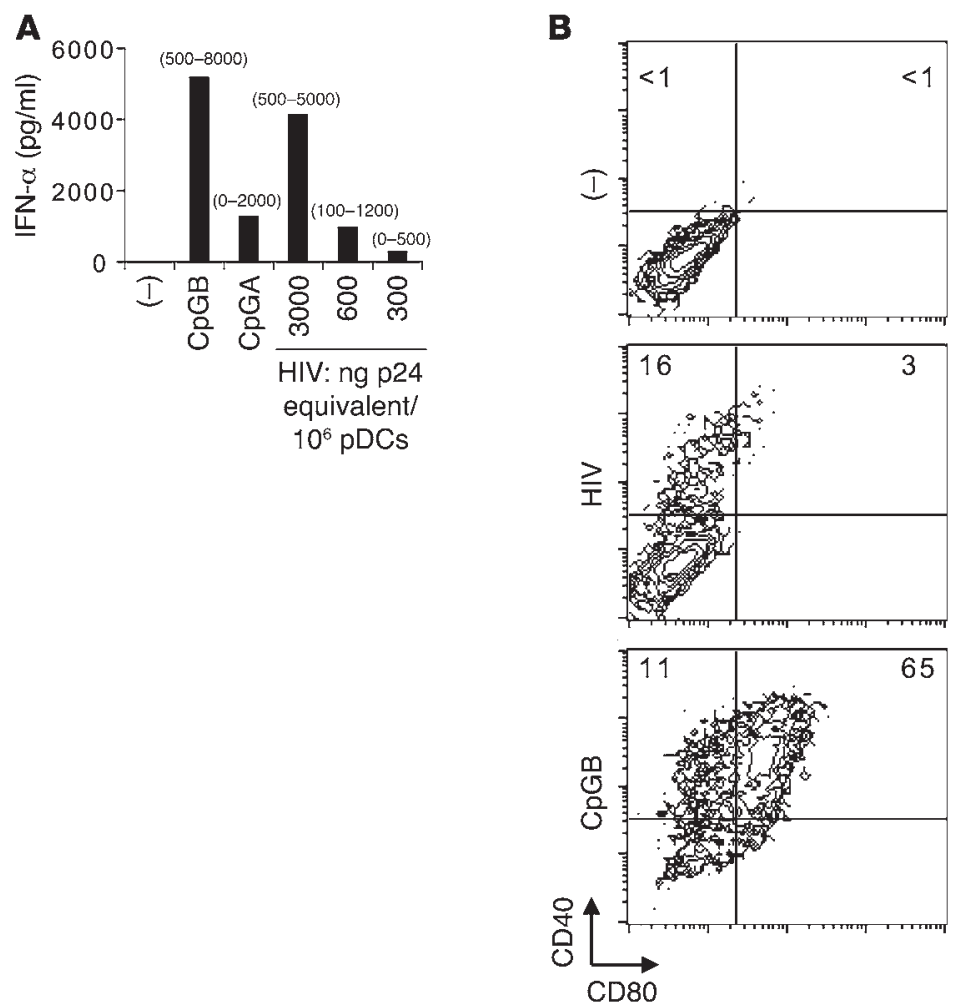
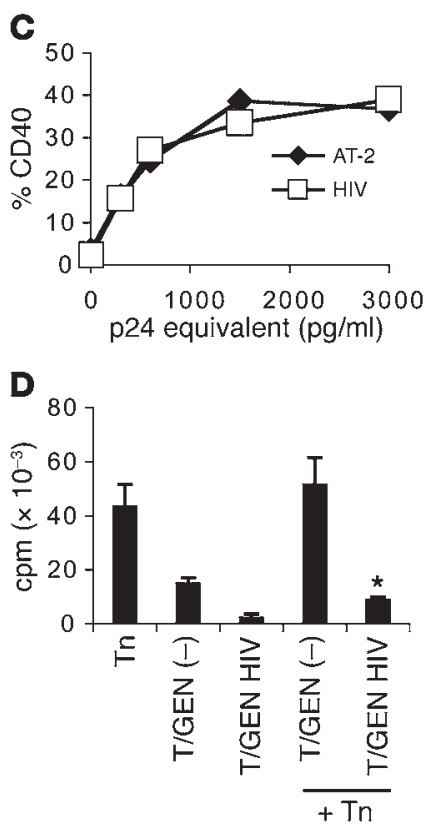

\section{Figure 2}

The leukemic cell line GEN secretes IFN- $\alpha$ and matures in response to HIV or AT-2 HIV. (A) Secretion of IFN- $\alpha$ by GEN cells upon stimulation with $2 \mu \mathrm{g} / \mathrm{ml} \mathrm{CpGB}, 20 \mu \mathrm{g} / \mathrm{ml} \mathrm{CpGA}$, or 300, 600, 3,000 ng/ml of p24 equivalent HIV. Ranges of IFN- $\alpha$ secretion in different experiments $(n>10)$ are indicated in brackets for each condition. (B) Maturation of GEN cells induced by HIV or CpGB, as measured by CD40 and CD80 upregulation. The percentages of cells in the upper quadrants are indicated. Data shown are representative of at least 8 experiments. (C) Dose-dependent maturation of GEN in response to HIV or AT-2 HIV, as measured by CD40 upregulation (representative of at least 8 experiments). (D) T cells stimulated by unactivated [T/GEN (-)] or HIV-activated GEN [T/GEN HIV] for 7 days were added to naive CD4+ T cells stimulated by anti-CD3/ anti-CD28. Proliferation was measured at day 5. ${ }^{*} P<0.01$, Student's $t$ test, compared with proliferation of naive $T$ cells alone.

\section{Discussion}

The status and role of Tregs in HIV infection is still debated. In models of retroviral infection in mice and cats (28-30) and in HIVinfected humans (31), certain populations of Tregs are involved in specific immune suppression. Indeed, removal of $\mathrm{CD} 4^{+} \mathrm{CD} 25^{+} \mathrm{T}$ cells in HIV-infected humans and SIV-infected macaques enhances antiviral immune responses as measured in vitro (32). On the other hand, Tregs could play a beneficial role during HIV infection by limiting general immune activation (33). Indeed, recent results have documented the accumulation of increased numbers of Tregs in lymphoid tissues, including gut-associated lymphoid tissues in HIV-infected humans and SIV-infected macaques $(34,35)$, where they could participate in dampening chronic $T$ cell activation.

How these Tregs are generated during the course of HIV infection is an unresolved question, but our data suggest that pDCs may participate in their differentiation from naive $\mathrm{CD} 4^{+} \mathrm{T}$ cells. We demonstrate here that HIV-pDCs can induce the differentiation of Tregs from naive $\mathrm{CD}^{+} \mathrm{T}$ cells, which can then suppress proliferative $\mathrm{CD}^{+} \mathrm{T}$ cell responses and DC maturation. IDO expression by $\mathrm{pDCs}$ is required for Treg differentiation and is induced in $\mathrm{pDCs}$ after endocytosis of HIV via CD4-gp120 interactions, followed by TLR7/ MyD88 triggering in acidic endosomal compartments.

Our results are in line with previous observations showing that pDCs can induce Treg differentiation when activated by viral stimulation, TLR triggering, or CD40-L engagement (7-10). As in these prior studies, we cannot formally exclude that the highly purified naive population contained some undetectable Tregs that expanded upon culture with activated pDCs. In a recent mouse tumor study, IDO-expressing pDCs from tumor-draining lymph nodes were able to activate resting Tregs from non-tumor-bearing mice (36) to suppress specific immune responses. Whether pDCs induce de novo differentiation or expansion of Tregs, the resulting population of Tregs displays a potent suppressive activity that can impact on $\mathrm{T}$ cell proliferation and DC maturation.

Previous studies have implicated ICOS-L, IL-10, and CTLA-4 in the differentiation or activation of Tregs by pDCs $(8,9,36)$, but blocking of these different pathways only induced partial (and minor) reversal of the suppressive activity, suggesting that synergistic and/or sequential activation of each of these pathways was responsible for the modest effects observed. Instead, the mechanisms responsible for Treg generation were shown to primarily involve IDO upregulation by pDCs, as demonstrated by siRNAmediated knockdown of IDO. IDO gene transcription is dependent on TLR7 and MyD88 signaling, indicating that HIV may signal through TLR7/MyD88 to induce transcription of the IDO gene. Further cognate interaction with activated or Tregs may induce a so-called "reverse signaling" (initiated by engagement of CTLA-4 with CD80/CD86 or CD40 with CD40-L) in pDCs to activate the 
A

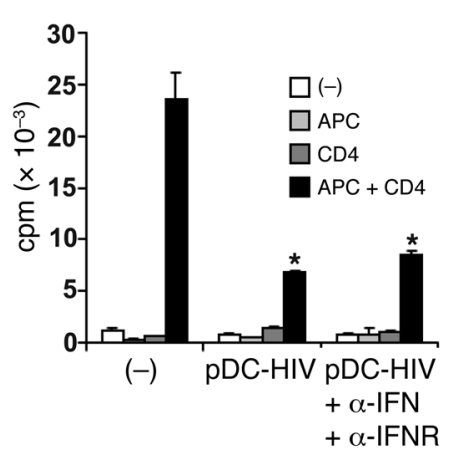

B

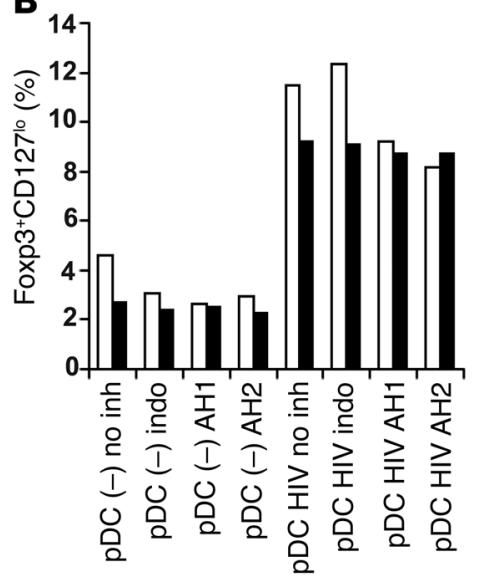

Figure 3

Mechanism of generation of Tregs. (A) Naive CD4+ $\mathrm{T}$ cells were stimulated as in Figure $1 \mathrm{~A}$, in the presence or absence of neutralizing antibodies against IFN- $\alpha$ and IFN- $\beta$ and IFN- $\alpha / \beta$ receptor ( $\alpha$-IFN $+\alpha$-IFNR). Responding T cells were added to a primary MLR as described in Figure $1 \mathrm{~A}$, and proliferation was measured after 5 days by thymidine incorporation. ${ }^{*} P<0.01$, Student's $t$ test, compared with proliferation of naive T cells. (B) Percentage of Foxp3 ${ }^{+C D} 127^{10}$ cells in $\mathrm{CD}^{+} \mathrm{T}$ cells stimulated by unactivated $(-)$ or AT-2 HIV-activated pDCs, in the presence of the EP2 and EP4 antagonists $\mathrm{AH} 6809(\mathrm{AH} 1)$ and $\mathrm{AH} 23848(\mathrm{AH} 2)$ or of the COX-2 inhibitor indomethacin (indo). Results of 2 of 4 experiments are shown as contiguous white and black bars for each condition. noncanonical NF-אB pathway (37-39) and induce full functional activity of IDO (40). IDO activity and tryptophan depletion may in turn induce GCN2 activation in T cells and Treg differentiation as part of a complex feedback loop (41). These pathways warrant further investigation of HIV-induced Treg generation by pDCs.

The fact that IDO expression by pDCs is implicated in Treg generation is compatible with a recent report showing that HIV- or AT2 HIV-stimulated pDCs express IDO, contributing to decreased $\mathrm{T}$ cell mitogenic responses by PBMCs from HIV-infected subjects (17). However, this study did not address the role of IDO in Treg generation. In some tumor models, IDO expression by tumor cells (42), or by pDC in the tumor-draining lymph node $(24,36)$, has also been shown to be responsible for induction or activation of Tregs. Our study adds virus infection as an additional trigger for IDO-dependent, pDC-mediated Treg differentiation.

In this study, we also demonstrate that $\mathrm{pDC}$-induced Tregs are able to suppress cDC activation by TLR agonists. The mechanisms responsible for the suppression are still to be uncovered. In recent studies of circulating $\mathrm{CD} 4{ }^{+} \mathrm{CD} 25^{+}$Tregs, several pathways have been implicated in the suppression of DC maturation. Thus, LAG-3 expressed by Treg can engage MHC-II on dendritic cells, inducing the recruitment of the phosphatase SHP-1 and dampening DC maturation (43). In another study, Tregs suppressed DC cytokine secretion in an IL-10-dependent fashion and inhibited DC phenotypic maturation in a contact-dependent fashion, but independently of TGF- $\beta$ and CTLA-4 (27). Recent work has also uncovered a role for the ectonucleotidase CD39 and CD73 expressed by Tregs in the generation of ATP metabolites (44), which suppress DC maturation through triggering of adenosine receptors (45). Investigation of the mechanism of Treg suppression of $\mathrm{cDC}$ maturation is currently under way.

It has previously been shown that Tregs can induce functional IDO expression in cDCs through CTLA-4 (46), providing another means by which pDC-induced Tregs could control the HIV immune response, that is, by inducing IDO expression in bystander DCs and generation of a suppressive microenvironment. The consequences of maturation suppression, therefore, can range from decreased presentation of HIV antigens or other microbial antigens to further induction of Tregs. The sum of these effects probably depends on the timing and place of cDCTreg encounter, as TLR-stimulated cDCs can secrete cytokines preventing Treg suppression (47), and Tregs themselves can be directly suppressed by TLR triggering (48). An interesting observation is that HIV can enhance survival of Foxp $3^{+}$Tregs in vitro (35), possibly through TLR7/8 triggering $(1,48)$, thereby potentially contributing to Treg perpetuation.

A recent study in SIV-infected macaques described increased mRNA levels of Foxp3 and CTLA-4 in secondary lymphoid organs and colon tissue, in association with increased IDO levels (34), suggesting the localization of Tregs expressing high levels of these transcripts. The lower circulating pDC numbers observed in human primary or chronic HIV infection may be due to recruitment of pDCs to mucosal or associated lymphoid tissues (3), where pDCs could prime naive T cells toward Th1 $(2,5)$ and Treg

Table 1

Mechanisms of Treg generation

\begin{tabular}{lcccc} 
Tregs induced by & Exp1 & Exp2 & Exp3 & Exp4 \\
T/pDC-AT-2 & $51 \pm 2.5$ & $39 \pm 1.7$ & $66 \pm 5.3$ & $35 \pm 2 \mathrm{~A}$ \\
T/pDC-AT-2 + anti-IL-10 & $36.7 \pm 3.2^{\mathrm{A}}$ & $30 \pm 1.3^{\mathrm{A}}$ & $\mathrm{ND}$ & $\mathrm{ND}$ \\
T/pDC-AT-2 + anti-GITR & $47 \pm 4.8$ & $39 \pm 3.7$ & $\mathrm{ND}$ & $35 \pm 3.3$ \\
T/pDC-AT-2 + anti-CTLA-4 & $25 \pm 2.9^{\mathrm{A}}$ & $23 \pm 1.6^{\mathrm{A}}$ & $\mathrm{ND}$ & $18 \pm 2.5^{\mathrm{A}}$ \\
T/pDC-AT-2 + anti-ICOS-L & $34 \pm 3.7^{\mathrm{A}}$ & $29 \pm 1.2^{\mathrm{A}}$ & $21 \pm 1.3^{\mathrm{A}}$ \\
T/pDC-AT-2 + anti-TGF- $\beta$ & $48 \pm 3$ & $42 \pm 3.7$ & $63 \pm 4.5$ & $\mathrm{ND}$ \\
\hline
\end{tabular}

Inhibition of Treg generation by blocking IL-10, GITR, CTLA-4, ICOS-L, and TGF- $\beta$ pathways. Naive CD4+ T cells were incubated with AT-2 HIV-stimulated pDCs (T/pDC-AT-2), in the presence of blocking antibodies, and tested for their suppressive activity as in Figure 1B. The ratio of naive T cells to Tregs was 1:3. The results of several experiments (Exp1-4) are shown, displayed as percentages of inhibition of proliferation ( \pm SD), as compared with proliferation without the addition of Tregs. AP $<0.05$, Student's $t$ test, compared with control values (T/pDC-AT-2 incubated with isotype control antibodies). ND, not determined. 
A

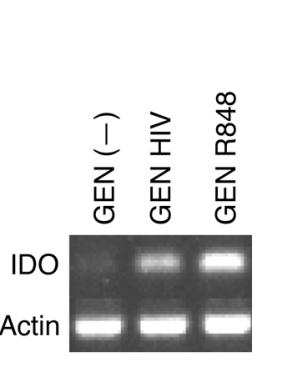

B

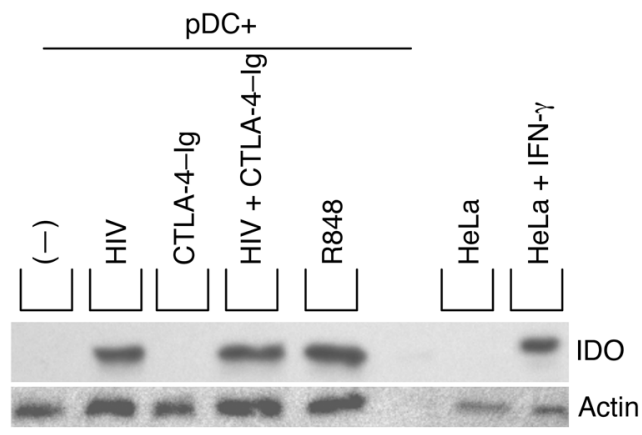

C

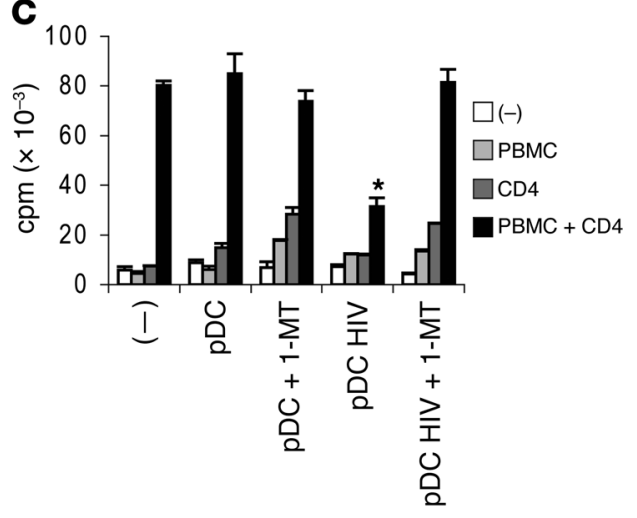

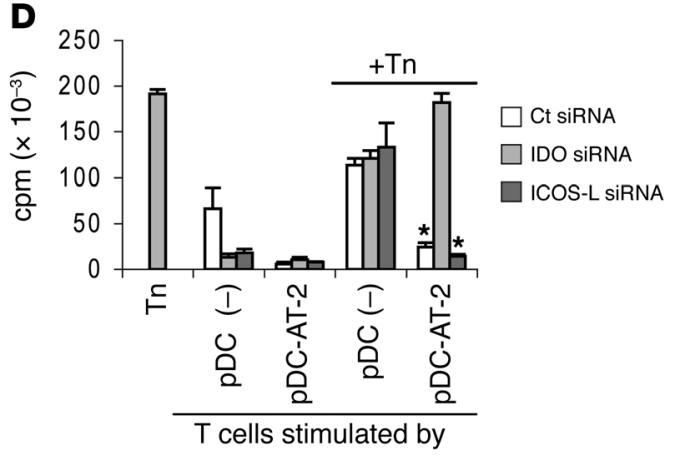

$\mathbf{F}$
$\mathbf{E}$

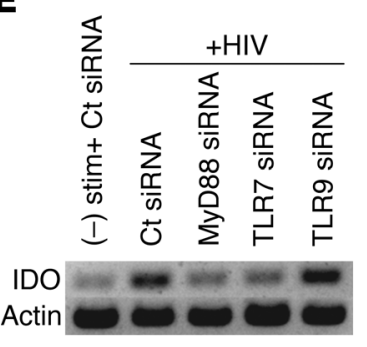

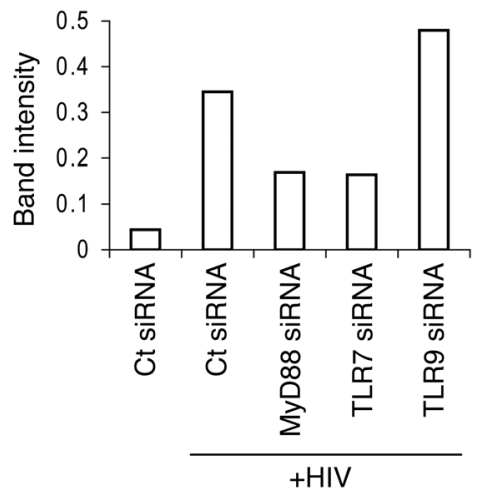

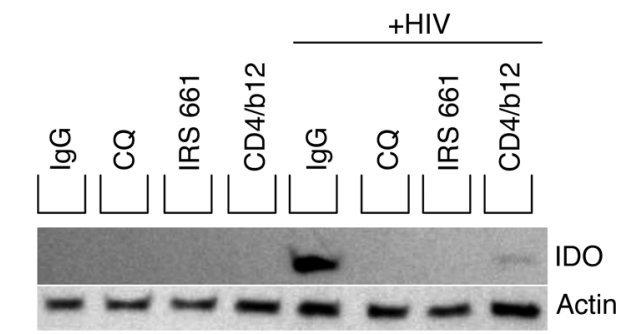

Figure 4

IDO-dependent generation of Tregs by AT-2 HIV-activated pDCs. (A) RT-PCR for IDO of GEN cells stimulated for 18 hours by HIV or resiquimod (R848). (B) Western blot for IDO of purified primary pDCs stimulated with HIV or resiquimod, in combination with CTLA-4-Ig. HeLa cells treated by IFN- $\gamma$ were used as a control. (C) Naive CD4+ $\mathrm{T}$ cells were stimulated by HIV-activated or unactivated pDCs as in Figure $1 \mathrm{~A}$, in the presence or absence of 1-methyl-tryptophan (1-MT), and then added to a primary MLR. Proliferation was measured at day 5 by thymidine incorporation. ${ }^{\star} P<0.01$, Student's $t$ test, compared with proliferation of naive T cells. Results are representative of at least 5 experiments. (D) GEN cells were electroporated with siRNA for IDO, ICOS-L, or control siRNA (Ct siRNA). After 1 day, they were stimulated by AT-2 HIV or left unstimulated and used as stimulating APCs for naive CD4+ $\mathrm{T}$ cells. After 7 days, the T cell suppressive activity was measured as in Figure 1B by adding the Tregs to naive CD4+ $\mathrm{T}$ cells (+Tn, 1:3 ratio). ${ }^{*} P<0.01$, Student t test, compared with proliferation of naive $T$ cells alone. (E) GEN cells were electroporated with siRNA for MyD88, TLR7, or TLR9 or control siRNA for 1 day, before stimulation with HIV. IDO transcripts were detected by RT-PCR after 6 hours. Right: Densitometric quantification of the IDO PCR product, normalized to the actin levels. (F) Purified primary pDCs were stimulated for 18 hours by HIV, with or without preincubation with chloroquine (CQ), IRS 661, neutralizing anti-CD4 and anti-gp120 antibodies (CD4/b12), or isotype control (IgG). IDO expression was monitored by Western blot analysis.

differentiation pathways. Importantly, our data are consistent with the concept that there is a dichotomy in the $\mathrm{PDC}$ response to HIV: production of proinflammatory factors to stimulate innate and adaptive antiviral immune responses (1) versus induction of pathways to limit an excessive response. These latter pathways may be critical for regulating the harmful chronic $T$ cell activation characteristic of progressive HIV disease. It will be important to examine pDC-specific transcripts for IDO within circulating or tissue-derived pDCs in HIV-infected humans and determine whether pDCs have the capacity to induce Tregs ex vivo. This information may help determine whether generation of Tregs by pDCs represents a significant factor in regulating HIV replication and DC maturation in vivo.

\section{Methods}

\section{Cells and reagents}

Cells. PBMCs were separated by density gradient centrifugation on a FicollHypaque (Amersham Biosciences) from leukocyte-enriched buffy coats obtained from the New York Blood Center. The pDCs were purified by 
A

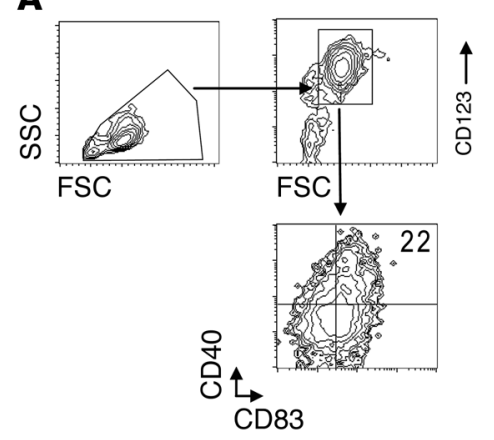

B

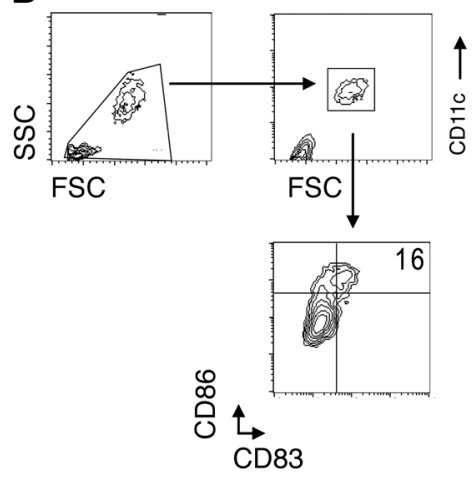

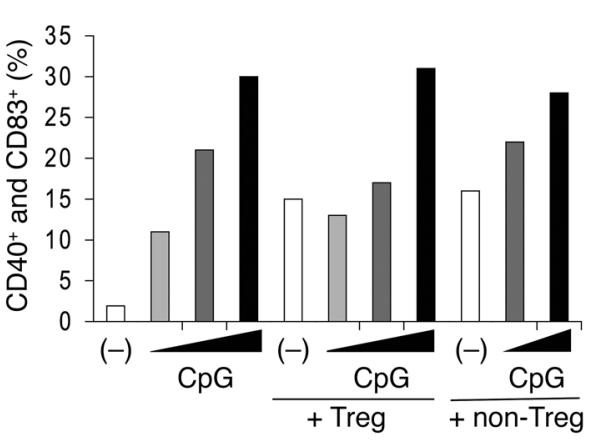

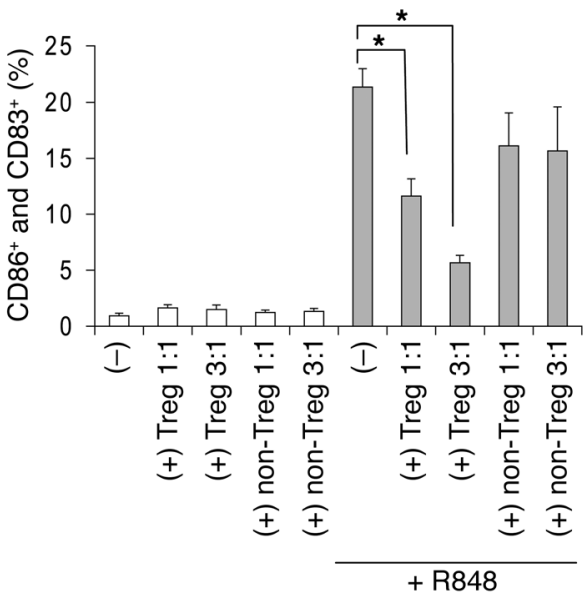

Figure 5

pDC-induced Tregs inhibit moDC maturation. Tregs generated by AT-2 HIV-activated pDCs were incubated for 24 hours with either pDCs (A) or moDCs (B). The DC subsets were then stimulated with $\mathrm{CpGB}(\mathbf{A})$ or resiquimod (B). (A) pDCs were stimulated with increasing doses of CpGB $(0,0.2,0.4,2 \mu \mathrm{g} / \mathrm{ml})$, and their expression of CD40 and CD83 was measured by flow cytometry after 1 day, in the gated CD123 ${ }^{\text {hi }}$ population (1 representative experiment of 3 is shown). The flow cytometry plot (left panel) shows cells stimulated by $2 \mu \mathrm{g} / \mathrm{ml}$ CpGB. The percentage of cells in the upper-right quadrant $\left(\mathrm{CD} 40^{+} \mathrm{CD} 3^{+}\right)$is indicated. (B) moDCs were incubated with different ratios of Tregs/moDCs ([-], 1:1, 3:1), and their expression of CD86 and CD83 was measured by flow cytometry by gating on CD11 $\mathrm{c}^{\text {hi }}$ cells. The percentage of cells in the upper-right quadrant $\left(\mathrm{CD} 86^{+} \mathrm{CD} 83^{+}\right)$is indicated. ${ }^{*} P<0.01$, Student's $t$ test. Results of 1 representative experiment of 3 are shown.
BDCA-4 magnetic bead separation (Miltenyi Biotec) as described previously (1), with a purity ranging from $70 \%$ to $98 \%$ (performed on buffy coats from at least 15 donors) (Supplemental Figure 4). Immunophenotyping of cells was done in PBS/0.1\% BSA (Sigma-Aldrich), and they were identified as BDCA-2 ${ }^{+}$ (Miltenyi Biotec) and CD123+ (BD Biosciences - Pharmingen) cells.

The pDC line GEN has been described previously (20) and was cultured with the MS-5 feeder cell line in RPMI 1640 GlutaMAX (Invitrogen) supplemented with sodium pyruvate, gentamicin, nonessential amino acids, and $10 \%$ FCS (Invitrogen).

Naive $\mathrm{CD}^{+} \mathrm{T}$ cells were purified in 2 steps from PBMCs. Naive CD $4^{+}$ $\mathrm{T}$ cells were enriched using the Naive CD4 Isolation Kit (Miltenyi Biotec). After enrichment, cells were stained and naive cells were sorted on a FACSAria (BD) as cells negative for CD8, CD56, CD11c, CD14, CD25, CD45RO, CD123, CD19, and HLA-DR and positive for CD45RA. The sorted cells were more than $98 \% \mathrm{CD}^{+}{ }^{+} \mathrm{CD} 45 \mathrm{RA}^{+}$.

moDCs were differentiated as previously described (2). Monocytes were purified from PBMCs by 2 hours of adherence and were cultured with $300 \mathrm{U}$ of IL-4/ml (Immunex) and $100 \mathrm{IU}$ of GM-CSF/ml (Schering-Plough) for 5 days to obtain monocyte-derived immature DCs.

Activating agonists. The type $\mathrm{B}$ CpG oligodeoxyribonucleotide type $\mathrm{B}$ (CPGB) $\left(T^{*} C^{*} G^{*} T^{*} C^{*} G^{*} T^{*} T^{*} T^{*} T^{*} G^{*} T^{*} C^{*} G^{*} T^{*} T^{*} T^{*} T^{*} G^{*} T^{*} C^{*} G^{*} T^{*} T\right.$, where asterisks indicate a phosphorothioate bond) was purchased from Integrated DNA Technologies and LPSs from E. coli (LPS-0127:B8) were from SigmaAldrich. Resiquimod (R848) was obtained from $3 \mathrm{M}$ and used at $10 \mu \mathrm{M}$.

Virus. Virions of HIV-1MN (X4-tropic, lot 3941) were produced from the HIV-1 CEMX174 (T1) cell line as previously described (1, 2). Briefly, virions were purified by sucrose gradient ultracentrifugation after cells were removed from harvested cell cultures by tangential flow filtration or by clarification. The virus-containing fractions, as determined by the UV profile and sucrose density, were pooled and centrifuged at 18,000 $\mathrm{g}$ (Beckman continuous flow rotor model CF32Ti) for 1 hour to pellet the virus.
The resulting viral pellet was resuspended in TNE buffer $(0.01 \mathrm{M}$ Tris- $\mathrm{HCl}$ [pH 7.2], $0.1 \mathrm{M} \mathrm{NaCl}$, and $0.1 \mathrm{mM}$ EDTA in Milli-Q water [Millipore]) to a final 1,000-fold concentration relative to the original volume of cell culture fluid. Aliquots were stored in liquid nitrogen vapor. Samples were titrated for the presence of infectious virus using AA2CL.1 cells and HIV-1 p24 antigen capture kits (AVP; National Cancer Institute). For preparation of noninfectious virus (AT-2 HIV-1, lot 3937) with functionally intact envelope glycoproteins, virus was inactivated by treatment with $1 \mathrm{mM} \mathrm{2,2'-}$ dithiodipyridine (AT-2; Sigma-Aldrich) and purified and concentrated like nontreated HIV-1 as previously described (49).

Inbibitors. 1-Methyl-DL-tryptophan (1-MT) was purchased from SigmaAldrich and used at a concentration of $200 \mu \mathrm{M}$. The neutralizing antibodies against human IFN- $\alpha / \beta$ receptor chain 2 (CD118), human IFN- $\beta$ (MMH-3), and human IFN- $\alpha$ (MMHA-2) were purchased from PBL InterferonSource and used at $10 \mu \mathrm{g} / \mathrm{ml}$. Neutralizing antibodies against TGF- $\beta 1$, IL-10, and GITR were purchased from R\&D Systems, while the antibody against CTLA-4 was from Ancell and that against ICOS-L was from eBioscience. They were all used at $10 \mu \mathrm{g} / \mathrm{ml}$. The EP2 and EP4 antagonists AH6809 and AH23848 were purchased from Cayman Chemicals, and the COX-2 inhibitor indomethacin was from Sigma-Aldrich. All were used at $25 \mu \mathrm{M}$.

\section{Treg generation and Treg assay}

Purified primary pDCs or the GEN cells were activated for 18 hours by 300 and $600 \mathrm{ng} / \mathrm{ml} \mathrm{p} 24$ equivalent AT2-HIV and extensively washed, before being added to allogeneic naive $\mathrm{CD}^{+} \mathrm{T}$ cells at a 1:20 ratio in $96-\left(2 \times 10^{5}\right.$ $\mathrm{T}$ cells $)$ or 24 -well $\left(2 \times 10^{6} \mathrm{~T}\right.$ cells $)$ plates for 7 days in complete medium. GEN cells were treated with mitomycin C (Sigma-Aldrich) before addition to the T cells. Staining for Foxp3 was performed using the Foxp3 staining kit from eBioscience according to the manufacturer's instructions. CD $25^{+}$ cells were purified using the $\mathrm{CD} 25^{+}$enrichment kit from Miltenyi Biotec according to the manufacturer's instructions. 
For the Treg suppression assay, Tregs generated during the culture with HIV-pDCs were mixed with $2 \times 10^{5}$ naive $\mathrm{CD}^{+} \mathrm{T}$ cells from the same donor in 96 round-bottom wells and stimulated either by antibodies against CD3 $(1 \mu \mathrm{g} / \mathrm{ml})$ and CD28 $(0.5 \mu \mathrm{g} / \mathrm{ml}$; BD Biosciences Pharmingen) or by irradiated PBMCs from the same donor as the $\mathrm{pDCs}$ used for Treg generation.

For Transwell cultures, Tregs were placed in the upper chamber of an HTS Transwell-96 $(0.4 \mu \mathrm{m})$ plate (Corning) and naive CD4 ${ }^{+} \mathrm{T}$ cells in the lower chamber, before stimulation by CD3 and CD28 antibodies.

\section{IDO detection}

RNA of cells was isolated using the RNeasy Mini Kit from QIAGEN using the manufacturer's protocol. The primers for the RT-PCR were: for IDO, 5'-GCGCTGTTGGAAATAGCTTC-3' (forward), 5' -CAGGACGTCAAAGCACTGAA- $3^{\prime}$ (reverse); and for $\beta$-actin, $5^{\prime}$-TGACCCAGATCATGTTTGAGA-3' (forward), ACTCCATGCCCAGGAAGGA-3' (reverse). The IDO protein was detected by Western blot analysis using rabbit polyclonal IDO antibody from AXXORA, and quantification of the band intensity was performed using ImageQuant TL software (Amersham Biosciences).

\section{siRNA electroporation}

GEN cells were electroporated with $1 \mathrm{nmol}$ siRNA at $900 \mathrm{~V}$ for $0.75 \mathrm{~ms}$ on a Gene Pulser Xcell (Bio-Rad) in OPTIMEM (Invitrogen), washed, and left in complete medium for 24 hours before stimulation by HIV.

TLR7 and ICOS- $L$ siRNA were purchased as ON-TARGETplus SMARTpool from Dharmacon and comprised 4 siRNA duplexes: INDO (coding for IDO), UCACCAAAUCCACGAUCAUUU (sense)/5'-AUGAUCGUGGAUUUGGUGAUU (antisense), UUUCAGUGUUCUUCGCAUAUU (sense) $/ 5^{\prime}$ UAUGCGAAAGAACACUGAAAUU (antisense), GUAUGAAGGGUUCUGGGAAUU (sense)/5'-UUCCCAGAACCCUUCAUACUU (antisense), GAACGGGACACUUUGCUAAUU (sense)/5'-UUAGCAAAGUGUCCCGUUCUU (antisense); TLR7, CAACAACCGGCUUGAUUUAUU (sense)/5'UAAAUCAAGCCGGUUGUUGUU (antisense), GGAAAUUGCCCUCGUUGUUUU (sense)/5'-AACAACGAGGGCAAUUUCCUU (antisense), GAAUCUAUCACAAGCAUUUUU (sense)/5'-AAAUGCUUGUGAUAGAUUCUU (antisense), GGAAUUACUCAUAUGCUAAUU (sense)/5'-UUAGCAUAUGAGUAAUUCCUU (antisense).

1. Beignon, A.S., et al. 2005. Endocytosis of HIV-1 activates plasmacytoid dendritic cells via Tolllike receptor-viral RNA interactions. J. Clin. Invest. 115:3265-3275.

2. Fonteneau, J.F., et al. 2004. Human immunodeficiency virus type 1 activates plasmacytoid dendritic cells and concomitantly induces the bystander maturation of myeloid dendritic cells. J. Virol. 78:5223-5232.

3. Hosmalin, A., and Lebon, P. 2006. Type I interferon production in HIV-infected patients. J. Lenkoc. Biol. 80:984-993.

4. Hoeffel, G., et al. 2007. Antigen crosspresentation by human plasmacytoid dendritic cells. Immunity. 27:481-492.

5. Cella, M., Facchetti, F., Lanzavecchia, A., and Colonna, M. 2000. Plasmacytoid dendritic cells activated by influenza virus and CD40L drive a potent TH1 polarization. Nat. Immunol. 1:305-310.

6. Rissoan, M.C., et al. 1999. Reciprocal control of $\mathrm{T}$ helper cell and dendritic cell differentiation. Science. 283:1183-1186.

7. Gilliet, M., and Liu, Y.J. 2002. Generation of human CD8 $\mathrm{T}$ regulatory cells by CD40 ligandactivated plasmacytoid dendritic cells. J. Exp. Med. 195:695-704.

8. Ito, T., et al. 2007. Plasmacytoid dendritic cells prime IL-10-producing T regulatory cells by inducible costimulator ligand. J. Exp. Med. 204:105-115.
TLR9. TLR9 siRNA was purchased from Dharmacon, and 2 siRNAs were pooled together: GACGGCAACUGUUAUUACAtt (sense)/UGUAAUAACAGUUGCCGUCtt (antisense), ACAAUAAGCUGGACCUCUAtt (sense)/ UAGAGGUCCAGCUUAUUGUtt (antisense).

ICOS- $L$ and $M y D 88$ and the negative control siRNA were purchased from Ambion: ICOS- $L$, GGUUACACUGCAUGUGGCAtt (sense)/UGCCACAUGCAGUGUAACCtc (anti-sense); MyD88, GGAGGAUUGCCAAAAGUAUtt (sense)/AUACUUUUGGAAUCCUCCtc (antisense), GCAAUUUAUACUUUACCCUtt (sense)/AGGGUAAAGUAUAAAUUGCtc (antisense), GGAGAUGGACUUUGAGUACtt (sense)/GUACUCAAAGUCCAUCUCCtc (antisense).

\section{Statistics}

Statistical analysis was performed on at least 3 independent experiments using 2-tailed Student's $t$ test. Statistical significance was defined as $P<0.05$. Results are presented as mean \pm SEM of triplicate wells where indicated.

\section{Acknowledgments}

This work was supported by grants from the Elizabeth Glaser Pediatric AIDS Foundation, the Bill and Melinda Gates Foundation, the Doris Duke Charitable Foundation, the Emerald Foundation, the Center for HIV/AIDS Vaccine Initiative (CHAVI; AI067854), and NIH grants AI0571278, AI044628, and AI061684. O. Manches is a Joan and Robert Arnow Fellow of the Cancer Research Institute. The content of this publication does not necessarily reflect the views of the Department of Health and Human Services, nor does mention of trade names, commercial products, or organizations imply endorsement by the US government.

Received for publication December 20, 2007, and accepted in revised form July 23, 2008.

Address correspondence to: Nina Bhardwaj, NYU Cancer Institute, New York University, 522 First Avenue, New York, New York 10016, USA. Phone: (212) 263-5814; Fax: (212) 263-6729; E-mail: Nina.Bhardwaj@nyumc.org.
9. Kawamura, K., Kadowaki, N., Kitawaki, T., and Uchiyama, T. 2006. Virus-stimulated plasmacytoid dendritic cells induce CD4+ cytotoxic regulatory $\mathrm{T}$ cells. Blood. 107:1031-1038.

10. Moseman, E.A., et al. 2004. Human plasmacytoid dendritic cells activated by $\mathrm{CPG}$ oligodeoxynucleotides induce the generation of $\mathrm{CD} 4+\mathrm{CD} 25+$ regulatory T cells. J. Immunol. 173:4433-4442.

11. Ochando, J.C., et al. 2006. Alloantigen-presenting plasmacytoid dendritic cells mediate tolerance to vascularized grafts. Nat. Immunol. 7:652-662.

12. Deeks, S.G., et al. 2004. Immune activation set point during early HIV infection predicts subsequent CD4+ T-cell changes independent of viral load. Blood. 104:942-947.

13. Hazenberg, M.D., et al. 2003. Persistent immune activation in HIV-1 infection is associated with progression to AIDS. AIDS. 17:1881-1888.

14. Wilson, C.M., Ellenberg, J.H., Douglas, S.D., Moscicki, A.B., and Holland, C.A. 2004. CD8+CD38+ T cells but not HIV type 1 RNA viral load predict CD $4+\mathrm{T}$ cell loss in a predominantly minority female HIV+ adolescent population. AIDS Res. Hum. Retroviruses. 20:263-269.

15. Munn, D.H., et al. 1998. Prevention of allogeneic fetal rejection by tryptophan catabolism. Science. 281:1191-1193.

16. Munn, D.H., and Mellor, A.L. 2007. Indoleamine 2,3-dioxygenase and tumor-induced tolerance.
J. Clin. Invest. 117:1147-1154.

17. Boasso, A., et al. 2007. HIV inhibits CD4+ T-cell proliferation by inducing indoleamine 2,3-dioxygenase in plasmacytoid dendritic cells. Blood. 109:3351-3359

18. Liu, W., et al. 2006. CD127 expression inversely correlates with FoxP3 and suppressive function of human CD4+ T reg cells. J. Exp. Med. 203:1701-1711.

19. Seddiki, N., et al. 2006. Expression of interleukin (IL)-2 and IL-7 receptors discriminates between human regulatory and activated T cells. J. Exp. Med. 203:1693-1700.

20. Chaperot, L., et al. 2006. Virus or TLR agonists induce TRAIL-mediated cytotoxic activity of plasmacytoid dendritic cells. J. Immunol. 176:248-255.

21. Sharma, S., et al. 2005. Tumor cyclooxygenase-2/ prostaglandin E2-dependent promotion of FOXP3 expression and CD4+ CD25+ T regulatory cell activities in lung cancer. Cancer Res. 65:5211-5220.

22. Mellor, A.L., and Munn, D.H. 2003. Tryptophan catabolism and regulation of adaptive immunity. J. Immunol. 170:5809-5813.

23. Mellor, A.L., and Munn, D.H. 2004. IDO expression by dendritic cells: tolerance and tryptophan catabolism. Nat. Rev. Immunol. 4:762-774.

24. Munn, D.H., et al. 2004. Expression of indoleamine 2,3-dioxygenase by plasmacytoid dendritic cells in tumor-draining lymph nodes. J. Clin. Invest. 114:280-290. 
25. Heil, F., et al. 2003. The Toll-like receptor 7 (TLR7)specific stimulus loxoribine uncovers a strong relationship within the TLR7, 8 and 9 subfamily. Eur. J. Immunol. 33:2987-2997.

26. Barrat, F.J., et al. 2005. Nucleic acids of mammalian origin can act as endogenous ligands for Toll-like receptors and may promote systemic lupus erythematosus. J. Exp. Med. 202:1131-1139.

27. Houot, R., Perrot, I., Garcia, E., Durand, I., and Lebecque, S. 2006. Human CD4+CD25high regulatory $\mathrm{T}$ cells modulate myeloid but not plasmacytoid dendritic cells activation. J. Immunol. 176:5293-5298.

28. Dittmer, U., et al. 2004. Functional impairment of CD8(+) T cells by regulatory T cells during persistent retroviral infection. Immunity. 20:293-303.

29. Iwashiro, M., et al. 2001. Immunosuppression by $\mathrm{CD} 4+$ regulatory $\mathrm{T}$ cells induced by chronic retroviral infection. Proc. Natl. Acad. Sci. U. S. A. 98:9226-9230.

30. Vahlenkamp, T.W., Tompkins, M.B., and Tompkins, W.A. 2004. Feline immunodeficiency virus infection phenotypically and functionally activates immunosuppressive $\mathrm{CD} 4+\mathrm{CD} 25+\mathrm{T}$ regulatory cells. J. Immunol. 172:4752-4761.

31. Kinter, A., et al. 2007. Suppression of HIV-specific $\mathrm{T}$ cell activity by lymph node $\mathrm{CD} 25+$ regulatory $\mathrm{T}$ cells from HIV-infected individuals. Proc. Natl. Acad. Sci. U. S. A. 104:3390-3395.

32. Legrand, F.A., et al. 2006. Strong HIV-1-specific T cell responses in HIV-1-exposed uninfected infants and neonates revealed after regulatory $\mathrm{T}$ cell removal. PLOS ONE. 1:e102.
33. Baker, C.A., et al. 2007. Peripheral CD4 loss of regulatory $\mathrm{T}$ cells is associated with persistent viraemia in chronic HIV infection. Clin. Exp. Immunol. 147:533-539.

34. Boasso, A., et al. 2007. Regulatory T-cell markers, indoleamine 2,3-dioxygenase, and virus levels in spleen and gut during progressive simian immunodeficiency virus infection. J. Virol. 81:11593-11603.

35. Nilsson, J., et al. 2006. HIV-1-driven regulatory T-cell accumulation in lymphoid tissues is associated with disease progression in HIV/AIDS. Blood. 108:3808-3817.

36. Sharma, M.D., et al. 2007. Plasmacytoid dendritic cells from mouse tumor-draining lymph nodes directly activate mature Tregs via indoleamine 2,3dioxygenase. J. Clin. Invest. 117:2570-2582.

37. Belladonna, M.L., et al. 2007. Immunosuppression via tryptophan catabolism: the role of kynurenine pathway enzymes. Transplantation. 84:S17-S20.

38. Grohmann, U., et al. 2007. Reverse signaling through GITR ligand enables dexamethasone to activate IDO in allergy. Nat. Med. 13:579-586.

39. Puccetti, P., and Fallarino, F. 2008. Generation of $T$ cell regulatory activity by plasmacytoid dendritic cells and tryptophan catabolism. Blood Cells Mol. Dis. 40:101-105.

40. Munn, D.H., Sharma, M.D., and Mellor, A.L. 2004. Ligation of B7-1/B7-2 by human CD4+ T cells triggers indoleamine 2,3-dioxygenase activity in dendritic cells. J. Immunol. 172:4100-4110.

41. Fallarino, F., et al. 2006. The combined effects of tryptophan starvation and tryptophan catabo- lites down-regulate $\mathrm{T}$ cell receptor zeta-chain and induce a regulatory phenotype in naive $\mathrm{T}$ cells. J. Immunol. 176:6752-6761.

42. Curti, A., et al. 2007. Modulation of tryptophan catabolism by human leukemic cells results in the conversion of CD25-into CD25+ T regulatory cells. Blood. 109:2871-2877.

43. Liang, B., et al. 2008. Regulatory T cells inhibit dendritic cells by lymphocyte activation gene- 3 engagement of MHC class II. J. Immunol. 180:5916-5926.

44. Borsellino, G., et al. 2007. Expression of ectonucleotidase CD39 by Foxp3+ Treg cells: hydrolysis of extracellular ATP and immune suppression. Blood. 110:1225-1232.

45. Novitskiy, S.V., et al. 2008. Adenosine receptors in regulation of dendritic cell differentiation and function. Blood. Online publication ahead of print. doi:10/1182/blood-2008-02-136325.

46. Fallarino, F., et al. 2003. Modulation of tryptophan catabolism by regulatory $\mathrm{T}$ cells. Nat. Immunol. 4:1206-1212.

47. Pasare, C., and Medzhitov, R. 2003. Toll pathway-dependent blockade of CD4+CD25+ T cellmediated suppression by dendritic cells. Science. 299:1033-1036.

48. Peng, G., et al. 2005. Toll-like receptor 8-mediated reversal of CD4+ regulatory $\mathrm{T}$ cell function. Science. 309:1380-1384.

49. Arthur, L.O., et al. 1998. Chemical inactivation of retroviral infectivity by targeting nucleocapsid protein zinc fingers: a candidate SIV vaccine. AIDS Res. Hum. Retroviruses. 14(Suppl. 3):S311-S319. 